Boise State University

ScholarWorks

1-1-2009

\title{
Diabetes Self-Management in a Latino Social Environment
}

Dawn Weiler

Boise State University

Janice D. Crist

University of Arizona 


\title{
Diabetes Self-Management in a Latino Social Environment
}

\author{
Dawn M. Weiler, PhD, APRN-ANP \\ Boise State University \\ Department of Nursing \\ Boise, ID \\ Janice D. Crist, RN, PhD \\ University of Arizona \\ College of Nursing \\ Tucson, AZ
}

\author{
Research Supported by: \\ Sigma Theta Tau/Western Institute of Nursing Research Grant \\ Endocrine Nursing Society Research Grant \\ Boise State University/Department of Nursing Faculty Research Grant
}

Financial Interest Disclosure: None

\begin{abstract}
Purpose:

The purpose of this qualitative descriptive study was to explore the socio-cultural influences and social context associated with living with type 2 diabetes among rural, migrant Latino adults.
\end{abstract}

\section{Methods:}

A qualitative descriptive study using grounded theory techniques was conducted. In-depth semistructured interviews were completed with ten participants ( 6 female and 4 male) ranging in age from 46-65 years and duration of diabetes diagnosis ranging from 1.5- 40 years.

\section{Results:}

An over-arching meta-theme Self Management in a Social Environment emerged. Every aspect of the process of self-management, as described in the four major themes, (1) Family Cohesion, (2) Social Stigma of Disease, (3) Social Expectations/ Perception of "Illness," and (4) Disease Knowledge and Understanding, was influenced by the social context.

\section{Conclusions:}

The familist traditions, central to the Mexican culture, had both positive and negative consequences on diabetes management. Tailoring clinical care and developing novel education approaches, to include family and community, is central to improving the health of this population. Recognizing and acknowledging the social stigma associated with diabetes, for this population, will promote understanding and improve clinician/patient communication. The socio-cultural influences that impact diabetes management practices (e.g., include family, in particular the primary female caregiver, and establish community and home based education sessions) must be integrated into clinical practice. Future research focused on population defined health and disease self-management, novel educational interventions, and family and community interventions focusing on the concept of "social stigma of disease" is indicated to further impact the health disparities of this population. 
The National Institutes of Health (NIH) define disparities in health as "differences in the incidence, prevalence, mortality, and burden of diseases and other adverse health conditions that exist among specific population groups in the United States". These disparities include shorter life expectancy, higher incidence and prevalence of disease states such as diabetes and cardiovascular disease, and higher infant mortality and mental disorder rates. Many factors can contribute to health disparities such as reduced access to health care, increased risk of morbidity and mortality due to occupation or hazard exposure; increased risk due to underlying biological, socioeconomic, ethnic and familial factors; influences of cultural values and level of education. ${ }^{1}$ The Latino population is one group with known health disparities, ${ }^{2}$ especially in the area of diabetes. ${ }^{3}$

Diabetes poses a significant public health challenge in the United States. It is estimated that 1.5 million new cases are diagnosed each year or 4,109 each day. ${ }^{4}$ Diabetes is a chronic disease, the prevalence of which has increased steadily over the past decade. Presently 14.6 million persons in the United States have been diagnosed with the disease while 6.2 million persons are estimated to have the disease but are undiagnosed. ${ }^{4}$ Diabetes is the sixth leading cause of death in the United States, primarily from diabetes related cardiovascular disease. Additionally, diabetes is the leading cause of non-traumatic amputations in the United States (82,000 each year or 225 each day), blindness among working-aged adults (22,000 each year or 60.2 each day), and end stage renal disease (44,400 each year or 122 each day). ${ }^{5}$

Diabetes is one of the fastest growing disease classifications within the United States, especially among the Latino population. ${ }^{4}$ Nationally 2.5 million or $9.5 \%$ of all Latinos, 20 years of age or older, have diabetes. Latino individuals are 1.7 times as likely to have diabetes compared to non-Hispanic white individuals of similar age. ${ }^{4}$ These numbers are on a steady increase and only account for diagnosed cases of diabetes. Prevalence rates for the common complications of diabetes, obtained from the Centers for Disease Control and Prevention 2002 and 2005 data for the Latino population, clearly imply that developing strategies for preventing the complications from diabetes is a critical need. For example, 16.5 per 100 Latino adults with diabetes have visual impairments; 430.4 per 100,000 Latino adults with diabetes have end-stage renal disease; and 26.9 per 100 Latino adults age 35 years or older with diabetes have self reported cardiovascular disease. ${ }^{4}$ Comparable figures for non-Hispanic white adults are 19.5, 262.7 , and 34.9 respectively. In addition, less than $60 \%$ of Latino adults with diabetes receive annual eye and foot exams, and participate in daily blood glucose monitoring. ${ }^{4}$

Among Mexican-American adults, a subgroup of Latino adults, type 2 diabetes has reached epidemic proportions with concomitant devastating health complications, morbidity and mortality. Fortunately, many of these complications could be prevented through self-management techniques that promote tight glucose control. The financial, physiologic and psychologic costs associated with this disease and its complications are extreme. The need to explore culturally congruent, cost reducing and health promoting disease self-management strategies is imperative for this high risk population. New insights gained from this exploration may provide a mechanism to improve health outcomes and decrease costs associated with this chronic disease and move away from the Latino belief that "diagnosis of diabetes is a death sentence" $6(\mathrm{p} 18)$ in the population.

Extensive research in diabetes care has been conducted; however, there is a significant knowledge gap related to the factors that influence the achievement of glycemic control and self-management practices of the Latino population, in general, and migrant workers specifically. Based on well-documented disparities in complications and health outcomes among Latino adults compared to Anglo-American adults, there is sufficient evidence to question whether traditional Anglo-American beliefs about self-management are successful or appropriate for the migrant Latino population. Traditional models view self-management as an individual responsibility. Whether this view is congruent with the collectivist cultural tradition held by many Latino adults is unclear. Equally unclear is the degree to which using traditional Anglo-American models of self-management, in teaching about managing type 2 diabetes, influences health outcomes in this population. Culturally congruent care and nursing interventions involves much more than an understanding of language and dietary preferences.

The purpose of this qualitative descriptive study was to explore the socio-cultural influences and social context associated with living with type 2 diabetes among migrant Latino adults. 


\section{Research Questions:}

This study was designed to answer the following research questions:

1) What are the socio-cultural influences that guide self-management practices among migrant Latino adults with type 2 diabetes?

2) How does the social context, including the ways in which family is viewed, influence the way in which migrant Latino adults conceptualize and enact diabetes self-management?

3) How do perception and experiences of migrant status and socio-economic status influence the way migrant Latino adults' self-manage type 2 diabetes?

\section{Method:}

A qualitative descriptive study using grounded theory techniques was conducted to provide a comprehensive summary of events in the everyday terms of those events. IRB approval was obtained from the The University of Arizona Human Subjects Protection Program.

In-depth semi-structured interviews were completed by the primary researcher with the assistance of one bilingual/bicultural translator, both trusted and readily accepted in the Latino community. Both the researcher and translator have worked with and in the population, both personally and professionally for 10+ years. Interviews were completed in the language preferred by the participants (Spanish $n=6$, English $n=4$ ) however, the translator was present at all interviews to translate as needed or requested. When requested by the participants, family members were also present during the interviews and provided additional insights on two occasions.

The primary research was experienced in semi-structured interviewing techniques and provided extensive training, including mock interviews, with the translator prior to initiating data collection. Debriefing was completed immediately following each interview by the researcher and translator and included evaluation of the interview process and techniques.

For interviews in Spanish:

1) Primary researcher asked the question in English.

2) The translator then asked the participant in Spanish.

3) The participant answered in Spanish (or English), if in Spanish.

4) The translator repeated the answer in English.

In this process, the audio recording and transcripts of the interview automatically contained a Spanish and English translation. Translation was completed for contextual meaning rather than simply verbatim. The contextual meaning of the words is more accurate than the words themselves. The translator and transcriptionist were given instructions regarding inference, wording, and phrasing, emphasizing the principle that translation for meaning promotes conceptual equivalence. ${ }^{7}$ Translators were asked to identify words that reflected different connotations or phrases that were awkward when back translated. ${ }^{8,9}$

Interviews were audio-recorded and transcribed by a single bilingual/bicultural transcriptionist who also confirmed accuracy of the "real-time" translations. Interview audio-recordings and transcripts were reviewed within 24 hours of the interview. Data was analyzed utilizing the process of constant comparative analysis using conventional content analysis strategies. Data analysis was completed by the two researchers independently, then jointly until consensus was achieved.

Trustworthiness of the research findings was achieved by adherence to the criteria of credibility, transferability, and confirmability as described by Lincoln and Guba. ${ }^{12}$ Throughout the research process, emerging categories and themes were confirmed with study participants and cultural experts to ensure the analysis was representative of their experiences and would be applicable to other similar groups in similar contexts. An audit trail of memos, notes, raw data and the reduction and interpretive products was maintained to allow others to identify without question how interpretations and research decisions were made. 


\section{Description of Sample}

Demographic data was collected on all study participants and analyzed quantitatively. In addition, a subset of questions from the Acculturation Rating Scale for Mexican Americans II (ARSMA-II) instrument, which measures cultural orientation toward the Mexican culture (MOS) and the Anglo culture (AOS) independently ${ }^{13}$ was utilized to obtain basic acculturation data (place of birth of participants and two preceding generations and language[s] spoken and read). This data was utilized only for the purpose of identifying breadth in the sample and providing descriptive statistics of the study participants.

Data saturation was reached with a total of 10 participants. ${ }^{12,13}$ Six female and 4 male participants were interviewed, ranging in age from 46-65 years (mean 56.5 years). The mean duration of diabetes diagnosis for the sample $(\mathrm{n}=10)$ was 10 years (range $1.5-40$ years). Six participants were married, one was divorced, and three were living with a partner but not married. The number of years participants were engaged in migrant farm work ranged from

$2-50$ years (mean 21.5 years). Three participants were no longer active farm workers due to disability $(\mathrm{n}=2)$ and finding other employment $(\mathrm{n}=1)$ with change in employment status occurring within the past 5 years (mean 2.7 years). Study participants reported living in the United States a mean of 32 years (range $2-65$ years). Thirty percent (all female) reported that they accessed healthcare services in Mexico. Seventy percent of the sample rated their income to pay for necessities as "almost enough" (30\%) or "never enough" (40\%). Participants reported frequency of returning to Mexico from "every 6 months" to "never" with the most common response being "every 1-2 years." Seventy percent of participants, $90 \%$ of their parents, and $83 \%$ of their grandparents were born in Mexico. Language(s) spoken in the home included 60\% "Spanish only" and 40\% both "Spanish and English." Language(s) read was $40 \%$ "Spanish only" and a $20 \%$ illiteracy rate was reported.

(Table 1 inserted here)

\section{Findings:}

An over-arching meta-theme Self Management in a Latino Social Environment emerged. Every aspect of the process of self-management, as described in the four major themes, (1) Family Cohesion, (2) Social Stigma of Disease, (3) Social Expectations/ Perception of "Illness," and (4) Disease Knowledge and Understanding, was influenced by the social context.

Family Cohesion, the first major theme to emerge from the interview data, is supported by the conceptual categories of "Surveillance," "Support," and "Family First." Family Cohesion is representative of the familist tradition of the Mexican culture. This theme emerged from the data as a central determinant of feeling supported; a motivator for diabetes self-management; and a potential stimulus for feelings of guilt when the health of self overshadowed the potential health risks of family members. Family members provided "surveillance" by monitoring, advising, and directing completion of diabetes management tasks which are illustrated in the following excerpts:

“...she's the one who's... You have to do this...And you have to do that and she's always on me...And she's always... You know... If it wasn't for her, I wouldn't be here now probably.” (M1)

“there doesn't go a day without somebody reminding me about, 'Oh...I haven't seen take your medicine today,' or 'I haven't seen you take your shot, your insulin' uh...somebody is always reminding me or asking if I did, uh...because they are always looking out for each other.” (M2)

“...mother was always looking after me that I eat right and take care of myself. Because I didn't give it much importance that I had diabetes.” (F4)

Overall, participants described the ever-present support provided to them, from both male and female family members, in the day-to-day process of managing their diabetes. When faced with unexpected and ongoing healthcare needs they turned to family for assistance. Family members (including nuclear, extended and kinship network members consistent with cultural definition of "family"), provided encouragement, direction and advice to participants in the process of disease management. Consistent with cultural norms, males and females tended to seek support from multigenerational family members of their same gender. 
One female participant, discussing how she delayed revealing her diabetes diagnosis to family members, is reflective of the category "family first." This participant was reflecting on her responsibility to inform her sisters that she had diabetes, and the need for them to also be evaluated for diabetes. Socio-cultural expectations of "family first" are illustrated in her excerpt:

“...how could I not [have] thought about it? I felt, how could you be so vain, I mean, so selfcentered. Because, it was 4 months and honestly I just was thinking about it and, oh my god! It just, like that. How could I not been thinking about them? [her sisters] How could I have been so in me? It was just like that, one morning I woke up and Oh my god!" (F1).

Participants described family as their primary motivator in disease management, commenting

"I have to be there for my family" (M2) and "don't want me to get deathly sick from that disease because it would hurt them [daughters of participant] (F3).

A father, holding his newborn infant, stated

“...this one came along, and I have to be-she's the one that's keeping me strong now... This is what's keeping me going” (M1).

The second major theme that emerged from the interview data was Social Stigma of Disease. Perceived social stigma when diagnosed with diabetes was emotionally devastating to many of the study participants resulting in denial and embarrassment. The social perception of individuals with diabetes as being "sick" appears to be the underlying foundation of the stigma surrounding diabetes. One participant stated

“...in my Hispanic culture I've seen it all the time, that anybody that had diabetes was just about the worst thing that there was, nothing compared to it, not seizures, not hypertension, nothing. Diabetes was a horrible monster; you were worthless if you had diabetes" (F1).

Another participant compared diabetes to having tuberculosis (TB), commenting that he was told as a child that TB was the worst thing but that this was not true; diabetes was.

Participant comments during the interviews often reflected on social perceptions of individuals with diabetes as being "sick." This appears to be the underlying foundation of the social stigma surrounding diabetes. Accordingly, Social Stigma of Disease ties directly into the next major theme of Social Expectations/Perception of "Illness."

Social Expectations/Perception of "Illness" emerged as the third major theme. Deep-seated social expectations that individuals with diabetes were by definition sick and unable to carry out normal activities emerged from the data. Comments reflected the perception that diabetes resulted in changes in personal appearance and energy level and doomed the individual to disabling outcomes (i.e., loss of limbs, heart disease, stroke, and "purple legs") and early death.

Additionally, participants expressed surprise when faced with the realization that "everyone knew" their diagnosis despite efforts to conceal this from family and community members. The following excerpt is reflective of this realization.

"Not Diabetes, no, and then I, and then it was a shameful thing, it was so shameful. And somehow, I didn't know it would go out to, spread out there to other people..." (F1).

The biggest challenge participants faced in the struggle to control their diabetes involved family and social (community) gatherings common in the Mexican culture. Participants reported that these gatherings revolved around the abundance of food and celebration. Non-attendance and/or declining food offers were disrespectful and socially unacceptable. When asked what the response would be if individuals brought healthy food choices with them to these events, participants reported this would be considered rude to the hosts and would not be accepted. Due to this conflict and the desire to better control their diabetes, frequency of family gatherings was decreased and the desire to "come up with better or new excuses" to not attend community fiestas was described. 
The fourth and final theme was Disease Knowledge and Understanding. Participants perceived their diabetes disease process knowledge as lacking. Study participants repeatedly expressed a desire for more education related to their diabetes. The interview data reflected that participants held a general understanding of the need to "eat healthy" but were unclear what this really meant. Participants expressed a need for specific guidance on how and what to cook, which they felt had not been adequately provided;

"they [healthcare providers] could have given classes on how to measure food ...food that I could eat. Tell my family what was going on. How they [family] could have helped me to take better care of ... diabetes. Give classes, really good classes on how to measure food and that kind of stuff, but they didn't do that" (F5).

Frustration with the lack of information provided was apparent in the interviews as exemplified by the following excerpt,

"they told me that I had to watch what I eat. It was the only thing that they tell us, they don't give us like a, you know sometimes they have a, some a, diets or something, they don't give us nothing" (M3).

One female participant stated

"the most difficult part is...uh...the cooking...you know how Mexican people cook, and it doesn't even come to mind what I should cook... and healthy" (F4).

Another made the following statement when discussing the education she received when she was diagnosed with diabetes;

"He said not to eat pork. Not to eat mango, or apples, carrots, and beets... a lot of food. He said that blood sugar was never gonna be controlled. It was be--gonna be going up all the time" (F5).

In addition, a lack of education regarding the disease process of diabetes emerged. Many participants described being informed, when diagnosed in Mexico, that if they did not control their blood glucose levels it would affect other organ systems (kidney, eye, heart). However, why and how this occurs was not a part of the information given.

Family presence at diabetes education sessions was minimal for those diagnosed in both the United States and Mexico. Participants commented that having family attend education classes with them would have been helpful in controlling their diabetes but stated that often this was not possible due to conflicting obligations, such as work schedules. When asked if other approaches to education would alleviate this barrier, many responded that bringing education classes to their home or community would be helpful;

"it would be beneficial for...uh...for everybody to come like...uh...the family, dad, brothers, sisters...that way they're all informed of what I can eat..." (F5).

One participant summed it up in the following statement

"not sure if he [partner] would come to...uh...out and to a class, but if, if somebody went to him...then he would say yes automatically" (F4).

Making education a family event was described in a favorable manner.

\section{Conclusions:}

This study revealed several socio-cultural influences that impact diabetes self-management practices for the migrant Latino adult which are congruent with familist cultural traditions. ${ }^{14-16}$ The familist traditions, central to the Mexican culture, were highly influential which is consistent with findings in previous studies. ${ }^{17-21}$ However, familism for migrant workers with diabetes had both positive and negative consequences on diabetes management. 
The social stigma coupled with the social expectations and perception of illness present in the social environment was highly influential on diabetes management practices. The over-shadowing social stigma associated with having diabetes resulted in embarrassment, shame, and devastation on the part of individuals diagnosed with diabetes. Denial and withdrawal from social situations were common, resulting in isolation and feelings of guilt due to having diabetes. These social responses, based on deep-seated social expectations of the physical results of diabetes (justified or not) resulted in self-doubt and shame. The impact of the social stigma in this population needs to be explored further. Extensive research has been completed on the topic of stigma ${ }^{20-22}$; however, little has been focused on the Latino population. The available literature directed at the Latino population is related to tuberculosis ${ }^{23,24}$ and mental illness. ${ }^{25}$ None were identified that focused on chronic illness in general, or diabetes specifically.

Stigma, as described by Goffman ${ }^{26}$ is a socially defined attribute that is deeply discrediting. These attributes are categories by which others are judged; "transforming them into normative expectations, into righteously presented demands". ${ }^{26(\mathrm{p} 2)}$ This definition is consistent with what emerged from the data in this study. The social expectations and associated social stigma, which the participants described, created a climate in which the participants' selfimage was confronted and affronted by the image others reflected back to them. In this process, shame emerges as individuals redefine the image they have of themselves. ${ }^{26}$ Changing socially defined category attributes and expectations is central to eradicating the stigma. ${ }^{21,27}$ The socially ascribed expectations of those individuals with diabetes are descriptive attributes of poor blood glucose control. In light of this, and the reported diabetes education deficiencies, improving diabetes disease process knowledge may be the route for ameliorating the stigma of diabetes in this population. This in turn would improve the health of this population through enhanced glucose control and the development of fewer complications.

\section{Limitations:}

The researcher's limited Spanish language skills must be considered a limitation of this qualitative study despite the skills and expertise of the bilingual, bicultural translator and transcriptionist utilized to enhance the truth value of the findings. Conducting cross-language and cross-cultural research increases the likelihood for the researcher to miss language and meaning nuances present in the data.

\section{Implications:}

The knowledge gained from this research can impact clinical care of this population. Clinicians must clarify with individual patients the extent of diabetes education they have received and promote options for further education as appropriate. Additionally, recognizing and acknowledging the social stigma associated with diabetes, for this population, will promote understanding and improve clinician/patient communication. Study findings provide rich information that can be used to tailor clinical care so that it is consistent with the socio-cultural influences that impact diabetes management practices (e.g., include family, in particular the primary female caregiver, and establish community and home based education sessions).

The findings of this study can be used to develop novel educational interventions. Shifting the education focus from the individual to the family and community may well be central to improving the health of this population. Additionally, exploring interventions to ameliorate the stigma associated with this very prevalent disease is essential. Reducing or eliminating the social stigma of diabetes would likely improve adherence to self-management practices and improve glucose control through the process of stress reduction and social acceptance.

Further exploration of the concept of "Stigma of Disease" is needed. It is anticipated that this concept is not unique to the Mexican population; however the precipitating factors leading to social stigma need to be investigated further. In addition, the rural context, and the potential influences this has on diabetes self-management, will provide further insights into the process of disease management in this population. The concept of illiteracy and its relationship to health, how this population defines health and disease self-management will provide the additional concepts needed for theory or model development. Once these concepts have been developed, the model or theory of diabetes selfmanagement for the Mexican migrant adult that emerges can be tested. 


\section{Acknowledgments}

The authors wish to express our deepest appreciation to our research participants for sharing their knowledge and to Linda R. Phillips, PhD, RN, FAAN (UCLA School of Nursing), Terry Badger, PhD, RN, FAAN and Sally J. Reel, PhD, APRN-FNP, FAAN, FAANP (University of Arizona College of Nursing) for your guidance and insights.

\section{References}

1. National Institutes of Health Web site. Health disparity defined. Available at: http://crchd.cancer.gov/definitions/defined.html. 2000. Accessed June 2007.

2. Laditka SB, Laditka JN, Drake BF. Home and community-based service use by older African American, Hispanic, and non-Hispanic white women and men. Home Health Care Serv Q. 2006;25(3):129-153.

3. Peek ME, Cargill A, Huang ES. Diabetes health disparities: a systematic review of health care interventions. Med Care Res Rev. 2007;64(5)(suppl):101S-156S.

4. Centers for Disease Control and Prevention (CDC). General information and national estimates on diabetes in the United States. Atlanta, GA: U.S. Department of Health and Human Services; 2005.

5. Centers for Disease Control and Prevention (CDC). General information and national estimates on diabetes in the United States. Atlanta, GA: U.S. Department of Health and Human Services; 2003.

6. Hakes D, Blanco J, Foxcroft B, Compean-Rincon J, Sanchez A. Health care access barriers for Idaho Latinos. Boise, ID: Idaho Primary Care Association; 2003.

7. Crist JD, Velazquez H, Durnan I, Figueroa DR. Intrument development of the confidence in home care services questionnaire for use with elders and cargivers of Mexican descent. Public Health Nurs. 2006; 23(3):284291.

8. Hilton A, \& Skrutkowski M. Translating instruments into other languages: development and testing processes. Cancer Nurs. 2002; 25(1):1-7.

9. Nelson McDermott MA, \& Palchanes K. A literature review of the critical elements in translation theory. Image J Nurs Sch. 1994; 26(2):113-117.

10. Lincoln YS, Guba EG. Naturalistic Inquiry. Beverly Hills, CA: Sage; 1985.

11. Cuellar I, Arnold B, Maldonado R. Acculturation rating scale for Mexican Americans-II: a revision of the original ARSMA scale. Hisp J Behav Sci. 1995;17(3):275-304.

12. Glaser BG. Theoretical Sensitivity: Advances in the Methodology. Mill Valley, CA: The Sociology Press; 1978.

13. Glaser BG, Strauss AL. The Discovery of Grounded Theory: Strategies for Qualitative Research. New York: Aldine De Gruyter; 1967.

14. Arce C. Dimensions of familism and familial identification. Paper presented at: National Conference on the Hispanic Family; 1978; Houston, TX.

15. Heller P. Familism scale: a measure of family solidarity. J Marriage Fam. 1970;32(1):73-80.

16. Weiler DM, Crist JD. Diabetes self-management in the migrant Latino population. Hisp Health Care Int. 2007;5(1):27-33. 
17. Crist JD. Mexican American elders' use of skilled home care nursing services. Public Health Nurs. 2002;19(5):366-376.

18. Ruiz ME. Familismo and filial piety among Latino and Asian elders: reevaluating family and social support. Hisp Health Care Int. 2007;5(2):81-89.

19. Strolin-Goltzman J, Matto H, Mogro-Wilson C. A comparative analysis of a dual processing substance abuse treatment intervention: implications for the development of Latino-specific interventions. Hisp Health Care Int. 2007;5(4): 162-168.

20. Carnevale F. Revisiting Goffman's stigma: The social experience of families with children requiring mechanical ventilation at home. J Child Health Care. 2007;11(1): 7-18.

21. Weiss M, Ramakrishna J, Somma D. Health-related stigma: rethinking concepts and interventions. Psychol Health Med. 2006;11(3): 277-287.

22. Scambler G. Sociology, social structure and health-related stigma. Psychol Health Med. 2006;11(3): 288-295.

23. Macq J, Solis A, Martinez G. Assessing the stigma of tuberculosis. Psychol Health Med. 2006;11(3): $346-352$.

24. McEwen MM, Boyle J. Resistance, health, and latent tuberculosis infection: Mexican immigrants at the U.S.Mexico border. Res Theory Nurs Pract. 2007;21(3):185-97.

25. Magana S, Garcia J, Hernandez M, Cortez R. (2007). Psychological distress among Latino family caregivers of adults with schizophrenia: the roles of burden and stigma. Psychiatr Serv. 2007;58(3): 378-384.

26. Goffman E. Stigma: Notes on the Management of Spoiled Identity. New York: Simon \& Schuster; 1963.

27. Heijnders M, Van Der Meij S. The fight against stigma: an overview of stigma-reduction strategies and interventions. Psychol Health Med. 2006;11(3): 353-363. 
TABLE 1. Participant Demographic Data by Gender

\begin{tabular}{|c|c|c|}
\hline & Male $(n=4)$ & Female $(n=6)$ \\
\hline \multicolumn{3}{|l|}{ Age } \\
\hline Mean & 57 & 56 \\
\hline Range & $46-64$ & $51-65$ \\
\hline \multicolumn{3}{|l|}{ Marital Status } \\
\hline Married & 3 & 3 \\
\hline Living w/Partner & 1 & 2 \\
\hline Divorced & & 1 \\
\hline \multicolumn{3}{|c|}{ Ability to Pay for Necessities } \\
\hline More than Enough & & 1 \\
\hline Just Enough & & 2 \\
\hline Almost Enough & 2 & 1 \\
\hline Never Enough & 2 & 2 \\
\hline \multicolumn{3}{|c|}{ Years Diabetes Diagnosis } \\
\hline Mean & 8.6 & 11 \\
\hline Range & $1.5-17$ & $2-40$ \\
\hline \multicolumn{3}{|c|}{ Years Living in United States } \\
\hline Mean & 34 & 30 \\
\hline Range & $19.0-46.0$ & $3-65$ \\
\hline \multicolumn{3}{|l|}{ Years Migration Work } \\
\hline Mean & 23 & 20 \\
\hline Range & $16-30$ & $2-50$ \\
\hline \multicolumn{3}{|c|}{ Access Healthcare in Mexico } \\
\hline Yes & 0 & 3 \\
\hline No & 4 & 3 \\
\hline
\end{tabular}

